\title{
Föderalstaatliche Traditionen und europäischer Handlungsbedarf
}

von Dieter Langewiesche

Historische Vorbilder föderalstaatlicher Entwicklungen können keine direkten Lösungen für den gegenwärtigen Handlungsbedarf der europäischen Politik aufzeigen. Gleichwohl bietet der Blick in die Geschichte die Möglichkeit, unterschiedliche Entwicklungspfade hypothetisch einzuschätzen und zu bewerten. Der vorliegende Beitrag fragt daher nach der Bedeutung des Föderalismus in den historischen Staatsbildungsprozessen in Europa sowie nach dem Verlauf der Identitätsbildung in Föderativstaaten, um deren Möglichkeiten und Grenzen herauszuarbeiten und auf denkbare Formen der europäischen Entwicklung zu beziehen. Im Ergebnis steht die Einschätzung, die Europäische Union könne in der Krise ihre eigene Alternative zur historisch harten Regel vom Krieg als Pfadbrecher und Pfadbildner gefunden haben. Zugleich erweisen sich vermeintliche Demokratiedefizite von historisch eher geringer Bedeutung, da Staatsbildungsprozesse fast ausnahmslos aus Elitenprojekten resultierten.

No direct solutions to the current European challenges can be derived from the history of federalism. However, a deeper look at past developments can be useful in hypothetically analysing and evaluating a number of potential future scenarios of further European integration. This contribution explains the importance of federalism regarding the origin of European statehood and outlines the process of identity formation in federal states to identify the apparent possibilities and limitations in view of potential future paths of European development. By harnessing the catalytic power of non-violent economic or political crises, the European Union may have overcome the historically hard correlation of war and state formation. At the same time, apparent deficits in democratic accountability should not be over-rated, as most historical processes of state formation were initiated by social and economic elites rather than popular demands.

\section{Einleitung}

Wer die Europäische Union als eine weltgeschichtliche Innovation bewertet, ${ }^{1}$ kann den gegenwärtigen europäischen Handlungsbedarf, den niemand bestreiten wird, nicht aus der Geschichte föderalstaatlicher Traditionen erschließen wollen.

1 Begründet u.a. bei Langewiesche, D.: Europäische Union - historische Vorbilder für eine staatspolitische Innovation?, in: ZSE 7/3-4 (2009), 348-363. 
Doch die Orientierungskraft der Geschichte erschöpft sich nicht im Studium historischer Vorbilder. Sie bietet kein Rezept für die Zukunftsgestaltung, aber doch einen Erfahrungsraum, der es ermöglicht, Gegenwartsprobleme angemessener einzuschätzen als ohne Selbstvergewisserung an der Vergangenheit. Deshalb soll in diesem Text erörtert werden, wie der Föderativstaat historisch zu verorten ist, welche Leistungen und Probleme mit ihm verbunden sind. Nur föderative Ordnungen oder Staatenverbindungen, die nicht auf Zentralisierung angelegt waren, bieten sich als historische Beobachtungsfelder an, die geeignet sein könnten, Entwicklungsmöglichkeiten der EU hypothetisch einzuschätzen. Denn so gestaltungsoffen die künftige institutionelle Form der EU auch ist, jede Annäherung an eine zentralstaatliche Gestalt dürfte seitens der Mitgliedstaaten und nach dem Mehrheitswillen ihrer Bürger ausgeschlossen sein. ${ }^{2}$ Die Bereitschaft, das historisch gewachsene staatliche Gehäuse und die Loyalitätsbindungen, die sich daran knüpfen, aufzugeben zugunsten einer Sache, die der eigenen Lebenswelt fern steht und sich deshalb in seinen Folgen nicht einschätzen lässt, bedürfte eines Ereignisses, das Vertrautes umstürzt und entwertet. Welche Art von Ereignissen haben in der Vergangenheit solche Wirkungen ausgelöst?

Um den Rückblick in die Geschichte von Föderativstaaten nicht in eine breite Erzählung ausufern zu lassen, werden zwei Fragen in den Mittelpunkt gerückt, die beide auf zentrale Gegenwartsprobleme der EU zielen:

- Was bedeutet „Föderalismus“ in den historischen Staatsbildungsprozessen in Europa? Denn um die Möglichkeiten und Grenzen von Staatsbildung geht es, wenn nach Entwicklungsperspektiven für die EU gefragt wird. Welche Prozesse sind erwünscht, welche nicht?

- Wie verlief die Identitätsbildung in Föderativstaaten? Hier soll vor allem erörtert werden, wie die Prozesse staatsbürgerlicher Identitätsbildung zwischen den staatlichen Ebenen - Einzelstaaten bzw. Länder, Kantone oder Regionen auf der einen Seite und Gesamtstaat auf der anderen - verbunden waren.

2 Vgl. dazu die Erörterungen von Patzelt, W. J.: Wie weiter mit Deutschland in der EU? Thesen zu den Gestaltungsaufgaben des Bundestages, in: ZSE 10/2 (2012), 241-266. 


\section{Föderalismus in europäischen Staatsbildungsprozessen}

Zunächst einmal gilt es festzuhalten: Der Übergang vom Staatenbund zum föderativen Bundesstaat ist historisch überall nur mit Gewalt gelungen, Revolutionsgewalt oder Kriegsgewalt, oft beides miteinander verbunden. ${ }^{3}$ Föderale Systeme entstanden keineswegs ,historisch als Assoziationen vormals unabhängiger Staaten", weil diese sich davon wirtschaftliche Vorteile und vermehrten außenpolitischen Einfluss versprochen hätten. ${ }^{4}$ Es war stets der Krieg, der die Sperren auf dem Weg in föderale Assoziationen von Staaten durchbrach. Auch der in der Politikwissenschaft gebräuchliche Begriff Pfadabhängigkeit verdeckt diese unangenehme historische Tatsache. So spricht Gerhard Lehmbruch, der die Pfadabhängigkeit in der Entwicklung des deutschen Bundesstaates immer wieder betont und plausibel begründet hat, von ,komplementären institutionellen Weichenstellungen" in den Jahren 1849, 1867/71, 1919 und 1945 bis 1949. ${ }^{5}$ Als Historiker wird man 1806 hinzufügen, als das Alte Reich endete und für diejenigen deutschen Staaten, die diese Zäsur und die staatliche Neuordnung Europas in der napoleonischen Ära überlebten, die lange Suche nach einer neuen Ordnung begann. Doch in allen diesen Fällen, ohne Ausnahme, war der Krieg der unverzichtbare Weichensteller: Sezessionskriege oder Integrationskriege und nicht selten beide miteinander verbunden. Das ist keine deutsche Besonderheit. Selbst der schweizerische Bundesstaat, der auf einer langen Tradition eidgenössischer Gemeinsamkeiten aufbauen konnte, ist aus einem Krieg zwischen den Kantonen hervorgegangen. Er verlief milde, doch es war ein Krieg, und es drohte eine Intervention der Habsburgermonarchie, die erst von der Revolution, die 1848

3 Näher ausgeführt bei Langewiesche, D.: Zentralstaat - Föderativstaat: Nationalstaatsmodelle in Europa im 19. und 20. Jahrhundert, in: ZSE $2 / 3$ (2004) 173-190; ders.: Recht und Revolution. Verfassungsstiftung durch Verfassungsbruch, in: Starck, Ch. (Hrsg.): Recht und Willkür, Tübingen, 2012, 27-45.

4 So jüngst Grasse. A.: Dissoziativer Föderalismus (2): Föderalismus in Italien, in: Härtel, I. (Hrsg.): Handbuch Föderalismus. Bd. IV: Föderalismus in Europa und der Welt, Heidelberg u.a., 2012, 797-817, 798. Grasse verweist hier auf das „Musterbeispiel USA“, das jedoch ein Musterbeispiel für die Staatsgründung und Staatsentwicklung durch Kriege ist. Das verkennt auch Ines Härtel, die den demokratischen Föderalismus mit den USA beginnen lässt, ohne deren Formung im Krieg zu erwähnen; vgl. Härtel, I.: Alte und neue Föderalismuswelten, in: Handbuch Föderalismus. Bd. I: Föderalismus als demokratische Rechtsordnung und Rechtskultur in Deutschland, Europa und der Welt, Heidelberg u.a., 2012, 3-22, 4. Auch die „doppelte Geburt des freiheitlich-demokratischen Föderalismus“ (ebd., 11) in Deutschland war jeweils eine Kriegsgeburt.

5 Lehmbruch, G.: Der unitarische Bundesstaat in Deutschland: Pfadabhängigkeit und Wandel, MPIfG Discussion Paper 02/2, Köln, 2002, 2. 
große Teile Europas erfasste, als Gefahr beseitigt wurde. ${ }^{6}$ Auch die USA ist als Bundesstaat im Sezessions- und Integrationskrieg, durchsetzt mit Bürgerkrieg, geschaffen worden. ${ }^{7}$ Die harte historische Regel lautet also: keine Transformation vom Staatenbund zum Bundesstaat ohne Krieg. Was man hier Pfadabhängigkeit zu nennen pflegt, ist stets aus dem kriegerischen Bruch mit dem bisherigen Geschichtspfad hervorgetrieben. Die Geburtsurkunde des Staates ist der Krieg. ${ }^{8}$ Darin macht der Föderativstaat keine Ausnahme.

Die Entwicklungsgeschichte der EU fügt sich ebenfalls in diese Regel, wenn wir, wie es üblich ist, den Zweiten Weltkrieg als kollektive europäische Ausgangserfahrung für den andauernden, in seinem Ziel offenen Einigungsprozess begreifen. Doch die weiteren Entwicklungsetappen kamen ohne die Veränderungsgewalt Krieg aus. Das ist weltgeschichtlich neu. Vor allem der bislang letzte große institutionelle Entwicklungsschub, ermöglicht von der Auflösung des Sowjetimperiums und dem Handlungsdruck, der davon ausging, ist historisch einzigartig. Noch nie in der bisherigen Geschichte hat sich ein Imperium ohne Krieg aufgelöst, noch nie hat es seine Peripherien ohne Krieg in die staatliche Souveränität entlassen, noch nie haben sich solche Sezessionsstaaten friedlich in ein anderes staatsähnliches Gebilde integriert und Teile ihrer neugewonnenen Souveränität abgetreten. All das ist seit 1990 in mehreren Schritten gelungen. ${ }^{9}$ Die Transformation der EU in diesem Prozess von Staatsauflösung und Staatsbildung - sie kommt wohl doch einer Neugründung gleich — gelang ohne die Transformationskraft Krieg. Das ist historisch gänzlich neu. Eine eiserne Geschichtsregel wurde durchbrochen.

Man muss dies vor Augen haben, um zu erkennen, dass der Begriff Pfadabhängigkeit hier eine neue Bedeutung erhält. Die EU, dieses begrifflich so schwer zu fassende Gebilde ohne Finalitätskonsens, hat sich fähig gezeigt, den staatlichen Umbruch im Europa der letzten beiden Jahrzehnte institutionell aufzufangen. Andreas Wirsching meint deshalb in seiner jüngst erschienenen „Geschichte

6 Knapp und präzise dazu Maissen, Th.: Geschichte der Schweiz, Baden, 2011, 196-199.

7 Als Überblick s. etwa Barney, W.L.: Battleground for the Union. The era of the civil war and reconstruction, 1848 - 1877, Englewood Cliffs, 1990. Generell zur Rolle des Bürgerkriegs vorzüglich $\mathrm{Ka}$ lyvas, S.N.: The Logic of Violence in Civila War, Cambridge, 2006.

8 In langer Perspektive dazu Reinhard, W.: Geschichte der Staatsgewalt. Eine vergleichende Verfassungsgeschichte Europas von den Anfängen bis zur Gegenwart, München, 1999; Porter, B.D.: War and the Rise of the State. The Military Foundations of Modern Politics, New York, 1994.

9 Umfassende Bilanz der Sezessionen und der Staatsbildungen, einschließlich der Nationalismen und Nationsbildungsprozesse im Prozeß der Auflösung der Sowjetunion bei Jahn, E. (Hrsg.): Nationalism in Late and Post-Communist Europe, 3 vol., Baden-Baden, 2008f. 
Europas in unserer Zeit", die EU habe ,gleichsam ihre eigene Pfadabhängigkeit entwickelt" ${ }^{10}$ und zwar eine, dies sei nochmals betont, für die es kein historisches Vorbild gibt. Wirsching sieht in der „Föderalisierung beziehungsweise Supranationalisierung“ den institutionellen Schlüssel für die Fähigkeit der EU, in der Krise Europas und durch sie einen eigenen Entwicklungspfad zu schaffen bzw. in der „Logik der ... Pfadabhängigkeit“ durch ,eine Politik des 'mehr Europa'“ fortzufahren. ${ }^{11}$ Die Krise als Pfadbildner. Von diesem Pfad abzuweichen, sei angesichts der „Folgekosten und des erheblichen Legitimitätsverlustes grundsätzlich nicht mehr möglich“. ${ }^{12}$ Hier läuft die vorzügliche zeithistorische Analyse wohl doch Gefahr, in politisch-pädagogische Prognostik zu münden. Jeder Pfadbruch vernichtet Legitimität und bürdet hohe Kosten auf. Dennoch ist die Geschichte voll davon. Und die Gegenwart ebenfalls.

„Föderalisierung beziehungsweise Supranationalisierung“ — dieses Ineinander von Begriffen, die nicht dasselbe meinen, findet sich auch in vielen Artikeln des jüngst erschienenen monumentalen „Handbuch Föderalismus“, in dem vornehmlich Juristen, Politologen und Ökonomen Entwicklungen und Probleme in unterschiedlichen Politikbereichen und Staaten (mit dem Schwerpunkt auf Deutschland und Europa) darlegen. ${ }^{13}$ Diese Begriffsannäherung, einhergehend mit einer kaum noch überschaubaren Vielfalt semantischer Differenzierungen von „Föderalismus" sind weitere Indizien dafür, dass man mit historischen Analogien vorsichtig sein sollte, wenn es um die Analyse der EU heute geht. Historisch wird man Föderalismus und Supranationalität strikt trennen müssen.

Föderalismus ist ein außerordentlich vielgestaltiger Begriff. Die juristischen und politologischen Artikel in dem erwähnten Handbuch bezeugen es erneut. Um nur einige der dort verwendeten Begriffe zu nennen: Neben dem administrativen Verbundföderalismus begegnet man einem Exekutivföderalismus, der dem Beteiligungsföderalismus gegenüber gestellt wird. Andere konfrontieren Trennungs- und Konsoziativföderalismus, kooperativen und wettbewerblichen Föderalismus, nichtstaatszentrierten gesellschaftlichen und staatszentrierten hoheitlichen Föderalismus, Reißbrett- und Flickenteppichföderalismus. Neben postmodernem Föderalismus ist vom Föderalismus sui generis und von klassischen Föderalstaaten oder auch von Überföderalisierung die Rede. Der Födera-

10 Wirsching, A.: Der Preis der Freiheit. Geschichte Europas in unserer Zeit, München, 2012, 190.

11 Ebd., 408.

12 Ebd., 18.

13 Härtel, I. (Hrsg.): Handbuch Föderalismus (4 Bde.), Heidelberg u.a., 2012. 
lismus, welcher der EU zugeschrieben wird, oszilliert begrifflich zwischen supranational und konsoziativ, Vollzugsföderalismus, föderalem Durchsetzungsverbund der Mitgliedstaaten und deren Einbindung in das föderale Mehrebenensystem einer Politikverflechtung, in der manche einen intergouvernementalen Staatenverbund, andere einen tendenziell bundesstaatlichen Verfassungsverbund erkennen.

Dieser Begriffswirrwarr spiegelt die Unbestimmtheit im Entwicklungsweg der EU wider, in den vor allem Juristen pfadbegrenzende Begriffspflöcke einzuschlagen hoffen, und ebenso äußert sich in dieser Begriffsunsicherheit die historische Vielfalt föderaler Gebilde. In dieser Situation könnte es hilfreich sein, sich daran zu erinnern, dass man früher für einen Staat, der mehrere Staaten umfasst, einen eigenen Begriff zur Verfügung hatte: zusammengesetzter Staat, ein Quellenbegriff, der lange Zeit nicht mehr verwendet wurde. Das 19. Jahrhundert kannte ihn noch. Der Schweizer Staatsrechtler Johann Caspar Bluntschli etwa verwendet ihn, vor allem an Samuel Pufendorf anknüpfend, in seiner Staatslehre, ${ }^{14}$ ebenso Friedrich Schleiermacher, der in ihm die „Cohäsionsgeselligkeit“ der Kleinen wirken sieht, während im Bundesstaat „die große Einheit dominirt" ${ }^{15}$ Als respublica mixta reicht dieses Begriffsfeld bis zu Aristoteles zurück, und in der Debatte über die Mischverfassung blieb es bis in die Gegenwart lebendig, wie Alois Riklin in einer grundlegenden Studie gezeigt hat. ${ }^{16}$ Ein großer Teil der frühneuzeitlichen Reichspublizistik hat das Alte Reich in diesem Sinne als „,ivitas composita“ charakterisiert. ${ }^{17}$ Erst in unserer Zeit ist es in die Wissenschaftssprache zurückgekehrt, nun aber zunächst in der heutigen lingua franca. Historiker sprechen für die frühe Neuzeit von composite state, composite monarchy, ${ }^{18}$ neuerdings findet auch der alte Quellenbegriff wieder Verwendung,

14 Bluntschli, J.C.: Deutsche Statslehre für Gebildete, Nördlingen, 1874, 142-149; ausführlich auf Pufendorf bezieht sich Bluntschli in ders.: Geschichte der Neueren Staatswissenschaft, Allgemeines Staatsrecht und Politik. Seit dem 16. Jahrhundert bis zur Gegenwart, 3. Aufl., München/Leipzig, 1881 (1. Aufl. 1864).

15 Schleiermacher. F.: Die Lehre vom Staat. Aus Schleiermacher's handschriftlichem Nachlasse und nachgeschriebenen Vorlesungen, hrsg. v. Chr. A. Brandis, Berlin, 1945, 27. Vgl. auch Rose, M.: Schleiermachers Staatslehre, Tübingen, 2011, 124-126.

16 Riklin, A.: Machtteilung. Geschichte der Mischverfassung, Darmstadt, 2006.

17 Vorzüglich dazu Stolleis, M.: Geschichte des öffentlichen Rechts in Deutschland. Erster Band 16001800, München, 1988; zu Pufendorfs Vorstellungen vom Reich unter 233-236; Burgdorf, W.: Reichskonstitution und Nation. Verfassungsreformprojekte für das Heilige Römische Reich Deutscher Nation im politischen Schriftum von 1648 bis 1806, Mainz, 1998; Kraus, H.-C.: Englische Verfassung und politisches Denken im Ancien Régime 1689 bis 1789, München, 2006, Kap. VI.

18 Vgl. Koenigsberger, H.G.: Dominium Regale or Dominium Politicum et Regale. Monarchies and Parliaments in Early Modern Europa, in: ders.: Politicians and Virtuosi. Essays in Early Modern Histo- 
allerdings meist begrenzt auf eine abgeschlossene Phase der Geschichte, nicht als systematischer Begriff, der auch für die Gegenwart nutzbar gemacht werden könnte. ${ }^{19}$ Doch der Sozialwissenschaftler Luis Moreno hat für Spanien jüngst die Begriffe „compound nationality“ und „compound national state“ vorgeschlagen $^{20}$, und die Imperienforschung spricht neuerdings von „conglomerate empires $^{\text {‘21 }}$ oder von „Kompositstaaten“'22.

Respublica composita, civitas composita, zusammengesetzter Staat - diese Begriffe, wissenschaftlich entwickelt vor allem für die frühe Neuzeit, verweisen darauf, dass die Fürsten zwar überall nach staatlicher Zentralisierung strebten, doch dieser Machtzentralisation blieben Grenzen gesetzt. In den hinzugewonnenen Gebieten wurden deren Rechte vielfach nicht angetastet. Dieses effiziente alteuropäische Ordnungsmodell geriet um 1800 unter Druck. Volksrevolution und Fürstenrevolution zielten in die gleiche Richtung: ein Generalangriff auf den zusammengesetzten Staat durch Zentralisierung staatlicher Macht, indem überkommene Zwischengewalten ausgeschaltet bzw. in ihren Selbstverwaltungskompetenzen stark beschnitten wurden. ${ }^{23}$ Ausgelöscht wurde das alte Ordnungsmodell jedoch keineswegs. In Europa überlebte es am stärksten in der

ry, London, 1986, 1-25; Elliott, J.H.: A Europe of Composite Monarchies, in: Past and Present 137 (1992), 48-71; Gustafsson, H.: Conglomerates or unitary states? Integration processes in early modern Denmark-Norway and Sweden, in: Fröschl, Th. (Hrsg.), Föderationsmodelle und Unionsstrukturen. Über Staatenverbindungen in der frühen Neuzeit vom 15. bis 18. Jahrhundert, Wien/München, 1994, 4562. Vgl. dazu ausführlicher Langewiesche, D.: Kleinstaat - Nationalstaat. Staatsbildungen des 19. Jahrhunderts in der frühneuzeitlichen Tradition des zusammengesetzten Staates, in: ders. (Hrsg.): Kleinstaaten in Europa. Symposium am Liechtenstein-Institut zum Jubiläum 200 Jahre Souveränität Fürstentum Liechtenstein 1806-2006, Schaan, 2007, 95-117, überarbeitet in: ders., Reich, Nation, Föderation. Deutschland und Europa, München, 2008, 194-210.

19 Becker, H-J. (Hrsg.): Zusammengesetzte Staatlichkeit in der europäischen Verfassungsgeschichte. Tagung der Vereinigung für Verfassungsgeschichte in Hofgeismar v. 19.3.-21.3.2001, Berlin, 2006 (darin Abhandlungen zu Böhmen, Niederlande, Russland, Großbritannien und Irland, Spanien, Habsburgermonarchie, Brandenburg-Preußen, Deutscher Bund).

20 Moreno, L.: Federalization in multinational Spain, in: Burgess, M./Pinder, J. (Hrsg.): Multinational Federations, New York, 2007, 86-107; ders.: Federalization and ethnoterritorial concurrence in Spain, in: Journal of Federalism 27/4 (1997), 65-84.

21 Gustafsson, H., a.a.O., 1994.

22 Leonhard, J.: Das Erbe der Vielfalt: die europäischen Empires und die Friedensverträge nach 1918, in: ZSE 10/3 (2012), 361-384, 364; der Autor geht nicht weiter auf die Begrifflichkeit ein. Eine aufschlußreiche gegenwartsbezogene Diskussion mit Blick auf Föderalismus und nationale Minderheiten bietet Kymlicka, W.: Minority Nationalism and Multinational Federalism, in: ders.: Politics in the Vernacular. Nationalism, Multiculturalism, and Citizenship, Oxford, 2001, 91-119.

23 Revolution und Monarchie werden meist als Gegensätze gesehen. Zu ihrem gewaltdurchsetzten Gemeinschaftswerk der Staatszentralisierung um 1800 s. Woolf, St.: Napoleon's integration of Europe, London, 1991; in zeitlich weiterer Perspektive bei Langewiesche, D.: Das Jahrhundert Europas. Eine Annäherung in globalhistorischer Perspektive, in: Historische Zeitschrift 296/1 (2013), 29-48. 
schweizerischen Eidgenossenschaft und in der Gestalt des Deutschen Bundes. Dass dieser Bund auf dem Wiener Kongress in der frühneuzeitlichen Tradition des zusammengesetzten Staates eingerichtet wurde, hat den deutschen Klein- und Mittelstaaten über ein halbes Jahrhundert lang Bestandsschutz gewährt. Der deutsche Nationalstaat von 1871 war dann kein zusammengesetzter Staat mehr, sondern ein föderativer.

Die früheren Formen von zusammengesetzter Staatlichkeit, die vor dem Zentralisierungsschub des 19. Jahrhunderts die Normalität waren, gewinnen aus der Gegenwartserfahrung zieloffener suprastaatlicher Entwicklungen eine neue Bedeutung. Ihre theoretische Durchdringung steht jedoch noch ganz am Anfang. Es dürfte aufschlussreich sein, die Institutionenordnung der EU in dieser Begrifflichkeit zu analysieren, um die Differenz, die historisch zwischen dem Föderativstaat und dem zusammengesetzten Staat besteht, für die Gegenwart nutzbar zu machen.

Ein solcher historisch informierter Blick auf die Gegenwart könnte helfen, irreführende Analogien zu vermeiden, weil er verdeutlicht, dass zusammengesetzte Staaten in Europa zwar die Normalität gewesen sind, jedoch nur in sehr wenigen diese Tradition seit dem 19. Jahrhundert in Föderalismus überführt worden ist. Dieser Schritt vom zusammengesetzten Staat zum föderativen Bundesstaat, wohlgemerkt stets ein kriegerischer Schritt, ist in Deutschland und in der Schweiz gelungen. Was dort heute Föderalismus bedeutet, folgt anderen Traditionen, anderen Entwicklungspfaden als in den meisten anderen Staaten Europas. Deshalb sei dafür plädiert, eine Begrifflichkeit zu entwickeln, die nicht höchst unterschiedliche historische Entwicklungen mit Blick auf die Gegenwart einebnet, wenn es darum geht, Zukunftsperspektiven für die EU zu entwerfen. Wer die Institutionenordnung der EU als föderativ charakterisiert und auf dieser Grundlage den Handlungsbedarf für die EU bestimmen will, sollte sich bewusst machen, dass man damit aus dem historischen Gedächtnis der meisten EU-Nationen aussteigt und zwischen ihnen konkurrierende Geschichtserfahrungen aufruft, die sich nicht leicht in gemeinsame Zukunftsvorstellungen übersetzen lassen.

Die Begriffsverschleifung von Föderalisierung und Supranationalisierung führt zu irrigen Selbstvergewisserungen an der Geschichte und leistet politischen Missverständnissen Vorschub. Kein historischer Staat in Europa war supranational. Wohl aber gab es viele Staaten, die mehrere Nationen umfassten. Aber daraus entstand keine Supranationalität, wenn man damit die Akzeptanz des Zusammenlebens mehrerer Nationen in einem Staat und eine darauf zugeschnittene Institutionenordnung meint. Die Nation hat sich historisch als eine außeror- 
dentlich machtvolle Zentralisierungskraft erwiesen. Supranationalität gehörte nicht zu ihrem Programm. Das Homogenitätsgebot, das der politischen Ordnungsidee Nation historisch eingestiftet ist, zielte stets auf kulturelle Angleichung an die Mehrheitsnation, also auf Unterordnung, oder aber auf staatliche Sezession, nicht hingegen auf eine supranationale Föderation von Gleichberechtigten. ${ }^{24}$ Diese historischen Bedeutungsprägungen sollte man bedenken, wenn man für die institutionelle Weiterentwicklung der EU von Föderalisierung oder Supranationalisierung spricht.

\section{Identitätsbildung in Föderativstaaten}

Es gehört heutzutage zu den gängigen Argumenten, eine volle Parlamentarisierung der europäischen Ebene setze voraus, dass die Legitimationsbasis der EU nicht mehr bei den „Völkern der Mitgliedstaaten“ liege, sondern beim „Volk in Europa“. Erst dann, so hat etwa der Politikwissenschaftlicher Winfried Steffani entlang dem Urteil des Bundesverfassungsgerichts von 1993 geschrieben, könne aus dem ,völkerrechtlichen EU-Vertrag eine europäische Verfassung“ werden. ${ }^{25}$ Solange das nicht der Fall ist, befinde sich die EU in einem DemokratieDilemma. Sie bleibe auf die Legitimierung durch die Verfassungsorgane der Mitgliedstaaten angewiesen. Ähnlich hat Dieter Grimm demokratietheoretisch gegen eine Verfassung für die EU argumentiert. ${ }^{26}$ Hier und jetzt, nicht grundsätzlich. Es fehle ihr heute, pointiert gesagt, eine europäische Staatsbürgergesellschaft. Die Verfassung könne sie nicht schaffen, sie bedürfe ihrer.

24 Es gab solche Vorstellungen, aber sie setzten sich nicht durch. Vgl. dazu Francis, E.: Ethnos und Demos. Soziologische Beiträge zur Volkstheorie, Berlin, 1965; zu den austromarxistischen Ideen (Otto Bauer, Karl Renner), die Habsburgermonarchie in einen Nationalitätenstaat zu verwandeln und zu den subkutanen wissenschaftsgeschichtlichen Wirkungen dieser Vorstellungen in der heutigen internationalen Nationsforschung s. Langewiesche, D.: „Die Sozialdemokratie hält die Nation für unzerstörbar und für nicht zerstörenswert“. Theoretische Reflexionen im Austromarxismus um 1900 und ihre Bedeutung für die heutige Nationalismusforschung, in: ders., Reich, Nation, Föderation, a.a.O., 2008, 93-110. Bluntschli hat versucht, die Schweiz als einen Nationalitätenstaat zu bestimmen. Allerdings ging er von einem einheitlichen Schweizer Volk aus, das aus drei (Sprach-)Nationalitäten bestehe. Bluntschli. J.C.: Allgemeines Staatsrecht. 1. Bd., München, 3. umgearbeitete Auflage, 1863, 83-85.

25 Steffani, $W .:$ Das Demokratie-Dilemma der Europäischen Union. Die Rolle der Parlamente nach dem Urteil des Bundesverfassungsgerichts vom 12. Oktober 1993, in: Demokratie in Europa: Zur Rolle der Parlamente, in: Zeitschrift für Parlamentsfragen, Sonderband zum 25jährigen Bestehen, hrsg. v. Steffani, W./Thaysen, U., Wiesbaden, 1995, 33-49, 48.

26 Grimm, D.: Braucht Europa eine Verfassung? In: JuristenZeitung 50/12 (1995), 581-632; erneut in: ders.: Die Verfassung und die Politik. Einsprüche und Störfälle, München, 2001, 215-254; s. dort auch: ders.: Ist die Zeit reif für eine europäische Verfassung?, ebd., 255-263; in historischer Längslinie, um zu prüfen, was staatliche Souveränität heute in Europa bedeutet: ders.: Souveränität. Herkunft und Zukunft eines Schlüsselbegriffs, Berlin, 2009. 
Nun sei nach historischen Analogien gefragt, um zu sehen, ob sich in ihnen föderativstaatliche Entwicklungs- und Handlungspotentiale gegenüber diesem Demokratie-Dilemma erkennen lassen. Der Blick wird vorrangig auf Deutschland als den größten EU-Bundesstaat mit langer föderativer Tradition gerichtet. Zu fragen ist: Wie war hier staatsbürgerlich-nationale Identitätsbildung mit den institutionellen Prozessen der Staatsbildung verbunden? Oder bezogen auf die Legitimität der politischen Ordnung: In welchem Verhältnis standen die deutschen Staatsvölker zum 'Volk in Deutschland'?

Zunächst gilt es generell festzuhalten: Ethnogenese folgt der Staatsbildung, nicht umgekehrt. Das hat die Mittelalterforschung für ganz Europa eindeutig gezeigt. ${ }^{27}$ Nicht das Volk schuf den Staat, sondern in den Staaten und mit ihnen entstanden die Völker Europas. Die nationalen Mythologien sehen das anders. Für sie ist das Volk etwas Überzeitliches, während Staaten kommen und gehen. Solche Mythen werden vor allem in Krisenzeiten handlungsrelevant. Darin liegt ein historisch bedingtes Handicap für die EU. Sie hat keinen Mythos, der in Krisenzeiten als Bindekraft handlungsrelevant werden könnte. Die Mythen der Völker in den Mitgliedstaaten sind allesamt national ausgerichtet.

Auch hier zeigt sich die EU als ein Zukunftslaboratorium ohne historisches Vorbild: ${ }^{28}$ ein suprastaatliches Gebilde, zu dessen negativem Identitätsfundament eine jahrtausendealte europäische Kriegsgeschichte gehört, die ihrerseits in einer Vielzahl von nationalen Mythen geronnen ist, in denen sich die Völker der EUStaaten gegeneinander abgegrenzt haben. ${ }^{29}$ Das europäische Mythenarsenal ist mit Feindbildern bestückt, es ist nicht auf Integration ausgerichtet. Nur die Zukunft kann zeigen, ob das neue Europa gemeineuropäische Mythen braucht, deren Fähigkeit zu vergesellschaften politischen Entscheidungsverfahren entzogen ist. Denn in dieser vorpolitischen Integrationskraft liegt die politische Be-

27 Hier sei nur auf die Schriftenreihe „Nationes“ (Sigmaringen) verwiesen, in der die Ergebnisse eines langjährigen mediävistischen Projektes präsentiert werden, und auch auf die Forschungen von Walter Pohl, u.a. Pohl, W. (Hrsg.): Der frühmittelalterliche Staat - europäische Perspektiven, Wien, 2009.

28 Zum Folgenden ausführlicher und mit der internationalen Fachliteratur Langewiesche, D.: Wozu Geschichtsmythen?, in: ders./Ivaničková, E./Mišková, A. (Hrsg.): Mythen und Politik im 20. Jahrhundert. Deutsche - Slowaken - Tschechen, Essen, 2013 (i.E.); ders.: Unschuldige Mythen: Gründungsmythen und Nationsbildung in Europa im 19. und 20. Jahrhundert, in: von Lingen, K. (Hrsg.): Kriegserfahrung und nationale Identität in Europa nach 1945. Erinnerung, Säuberungsprozesse und nationales Gedächtnis, Paderborn, 2009, 27-41.

29 Den besten Einblick in die nationalen Mythen Europas bieten die Begleitbände zu zwei Ausstellungen des Deutschen Historischen Museums Berlin: Flacke, M. (Hrsg.): Mythen der Nationen: ein europäisches Panorama, München/Berlin, 1998, 2. Aufl. 2001; dies. (Hrsg.): Mythen der Nationen. 1945 - Arena der Erinnerungen. 2 Bände, Berlin, 2004. 
deutung von Mythen. Bisher haben offensichtlich alle Nationalstaaten nationale Mythen als gesellschaftliche Bindekraft gebraucht. Ob das Europa der EU solche Mythen ebenfalls benötigen wird und sie friedlich hervorbringen kann, ist offen. Bislang galt stets und überall: die Hoch-Zeit des Mythos ist der Krieg. Er steht im Zentrum der Mythen aller Nationen. Wenn aus EU-Krisen wie der gegenwärtigen friedlich ein genuin europäischer Mythos hervorgehen sollte, so wäre das, legt man als historische Analogie die Rolle der Mythen in den Nationalgeschichten zugrunde, sicherlich hilfreich für den langen Weg von den Völkern in Europa zu einem Volk in Europa. Die Krise würde auch hier als gemeinschaftsbildende Kraft an die Stelle des Krieges treten.

Zurück zur Frage nach dem Verhältnis von Identitäts- und Staatsbildung in Deutschland. Aufschlussreich ist, wie dieses Verhältnis von der Präambel des Grundgesetzes bis zu ihrer grundlegenden Revision im Jahre 1990 bestimmt worden ist: „Das gesamte Deutsche Volk bleibt aufgefordert, in freier Selbstbestimmung die Einheit und Freiheit Deutschlands zu vollenden. ${ }^{630}$ Welche Einheit der Deutschen ist hier gemeint? Die reale Geschichte der deutschen Nation kann es nicht sein. Die staatlich-nationale Einheit der Deutschen, der die alte Grundgesetz-Präambel mythisch Ewigkeit stiftet ${ }^{31}$, hatte nur ein Dreivierteljahrhundert gedauert, 1871 bis 1945, ein Wimpernschlag in der Geschichte der deutschen Nation. Und selbst in dieser Zeit lebten die Deutschen zweistaatlich, wenn man die lange Geschichte der deutschen Nation, zu der stets auch Österreich gehört hatte, als historischen Maßstab nähme.

In der deutschen Nationalgeschichte, ebenso in der schweizerischen, gingen Nation und Staat sehr lange getrennte Wege. Sie zusammenzuführen in der Gestalt eines gemeinsamen Nationalstaates wurde in Deutschland erstmals in der Revolution 1848/49 versucht und dann, mit Erfolg, 1871. Früher nicht. Deshalb war die Vorstellung von deutscher Nation von Beginn an vielstaatlich-föderal

30 Im Internet mit allen Änderungen im zeitlichen Verlauf: http://www.verfassungen.de/de/gg/grundgesetzvergleiche-i.htm.

31 Am klarsten hat das der Philosoph Kurt Hübner herausgearbeitet, dem auch wichtige Studien zur Erkenntnisfunktion des Mythos zu verdanken sind: Hübner, K.: Die Wahrheit des Mythos, München, 1985; ders.: Das Nationale, Graz u.a., 1991. Vgl. insbesondere Eliade, M.: Kosmos und Geschichte. Der Mythos der ewigen Wiederkehr, Frankfurt a.M., 2007 (erstmals 1949 im französischen Original); ders.: Myth and Reality, Long Grove, 1963. Zum Geschichtsmythos der Grundgesetz-Präambel, zu ihrer Entmythisierung 1990 und zum Mythenverzicht in der EU s. Langewiesche, D.: Verfassungsmythen und ihr Ende. Die Präambeln des Grundgesetzes der alten und neuen Bundesrepublik Deutschland und des Verfassungsentwurfs der Europäischen Union im Vergleich, in: ders.: Zeitwende. Geschichtsdenken heute, hrsg. v. Buschmann, N./Planert, U., Göttingen, 2008, 135-143. 
ausgerichtet, zunächst im Heiligen Römischen Reich deutscher Nation und dann im Deutschen Bund. Der deutsche Nationalstaat von 1871 überführte diese Vielstaatlichkeit der deutschen Nation erstmals in eine bundesstaatliche Gestalt. Die Ordnungsidee deutsche Nation verkraftete diesen Bruch mit der historischen Tradition, denn sie verstand sich als eine Föderativnation ${ }^{32}$. Ganz anders als etwa in Frankreich.

Bis 1870 haben sehr viele Deutsche diesen deutschen Zentralstaat in seiner preuBisch-protestantischen Dominanz nicht gewollt. Vor allem im deutschen Süden und Südwesten nicht, aber nicht nur dort. Erst der Krieg, und nur er, durchbrach das jahrhundertealte Gehäuse der deutschen Vielstaatlichkeit und überführte es in einen Bundesstaat, dessen Legitimität zunächst keineswegs gesichert schien. Weder in der Gesellschaft noch bei seinen Fürsten. Den ersten Schritt aus dem deutschen Geschichtspfad, den im weiten Spektrum zwischen zusammengesetztem Staat und Bund begrifflich präzise zu verorten zu allen Zeiten schwer gefallen ist ${ }^{33}$, erzwang ein innerdeutscher Krieg, der 1866/67 Sezession (Österreich) und staatliche Integration (Norddeutscher Bund) mit Staatsvernichtung durch Annexionen Preußens verband. Den zweiten Schritt ermöglichte der Einigungskrieg gegen Frankreich, aus dem das Deutsche Reich hervorging. Wie sich ohne diesen Krieg die Beziehungen zwischen dem preußischen Norden und den anderen Teilen Deutschlands entwickelt hätten, kann niemand wissen.

Dass dieser neue deutsche Bundesstaat, den Zeitgenossen als Ergebnis einer militärisch geführten „Revolution von oben“ verstanden, so rasch gesellschaftliche Legitimität errang, obwohl er doch mit der Grundlinie deutscher Geschichte, ihrer Vielstaatlichkeit, brach, hatte vor allem drei Gründe - freilich nur unter kräftiger Pointierung, um Analogieperspektiven zur EU zu öffnen:

- Es gab seit langem die Vorstellung von einer deutschen Nation, welche die staatliche Vielheit ideell umfasst, und in der ersten Hälfte des 19. Jahrhunderts entstand in der Gesellschaft der Wunsch nach nationaler Einheit. Wie

32 Zum Begriff der Föderativnation s. Langewiesche, D./Schmidt, G. (Hrsg.): Föderative Nation. Deutschlandkonzepte von der Reformation bis zum Ersten Weltkrieg, München, 2000.

33 Zur heutigen Debatte s. Schönberger, C.: Die Europäische Union als Bund. Zugleich ein Beitrag zur Verabschiedung des Staatenbund-Bundesstaat-Schemas, in: Archiv des öffentlichen Rechts, 129/1 (2004), 81-120; Möllers, C.: Der vermisste Leviathan. Staatstheorie in der Bundesrepublik, Frankfurt/M., 2008; Isensee, J.: Europäische Nation? Die Grenzen der politischen Einheitsbildung Europas, in: Decker, F./Höreth, M. (Hrsg.): Die Verfassung Europas, Wiesbaden, 2009, 254-280; zur Kritik an diesen Positionen Langewiesche, D., Europäische Union - historische Vorbilder, a.a.O., 2009; zur Begriffsgeschichte grundlegend Koselleck, R.: Bund, Bündnis, Föderalismus, Bundesstaat, in: Geschichtliche Grundbegriffe, Bd. 1, Stuttgart, 1972, 582-671. 
sie als Staatsordnung gestaltet werden sollte, veränderte sich, und es blieb strittig. Die Vorstellungen reichten vom Staatenbund über den föderativen Bundesstaat bis hin zum Zentralstaat, der die Einzelstaaten auslöscht. Letzteres wäre in Deutschland - anders als in Italien - wohl nur in republikanischer Gestalt, erzwungen durch eine antimonarchische Revolution, möglich gewesen. Als der Krieg den Weg in den monarchischen Bundesstaat öffnete, erwies sich die ordnungspolitisch offene Leitidee deutsche Nation als eine starke Kraft für die gesellschaftliche Akzeptanz des neuen Bundesstaates. Sich auf die Nation berufen zu können, stiftete Legitimität, die auch diejenigen anerkannten, die gegen diesen Staat ideell und mit der Waffe gekämpft hatten. Über eine solche in der Geschichte verankerte, zu Mythen geronnene Einheitsvision verfügt das EU-Europa nicht. Die heute vielbeschworene europäische Wertegemeinschaft ist ein Zukunftsprojekt, wie der deutsche Bundespräsident jüngst in seiner Rede über die Perspektiven der europäischen Idee zu Recht hervorgehoben hat, verankert in Verträgen und Gesetzen, und dennoch ein „Versprechen ${ }^{\text {‘34 }}$, kein historisch erprobtes Fundament. Wer die europäische Wertegemeinschaft hingegen in der Vergangenheit angelegt sieht, wird sich der Einsicht nicht entziehen können, dass in ihr jahrhundertelang gegeneinander Kriege geführt und von den Völkern und Nationen scharfe Trennlinien gezogen worden sind.

Der Blick zurück zeigt aber zugleich, dass die Entstehung von politischer Identität nicht an die vorgängige Demokratisierung der politischen Ordnung gebunden war. In der deutschen Geschichte und ebenso in der Geschichte der anderen EU-Staaten blieb die Vision einer staatlichen Einheit der Nation lange Zeit ein Elitenprojekt. Scharf zugespitzt: die breite gesellschaftliche Legitimation ging der nationalen Staatsbildung nicht voraus, sie wuchs innerhalb der neuen Staaten heran. Der Weg vom Elitenprojekt zur Akzeptanz durch die breite Bevölkerung vollzog sich in einem konfliktreichen Prozess. Deshalb sei vorgeschlagen, in der Tatsache, dass der bisherige EUStaatsbildungsprozess immer noch vorrangig ein Elitenprojekt ist, nicht per se ein dauerhaftes Demokratie-Dilemma angelegt zu sehen.

- Die gesellschaftliche Akzeptanz des Elitenprojekts deutscher Nationalstaat wurde durch den Föderalismus erleichtert. Er ermöglichte es, eine Vielzahl einzelstaatlicher Institutionen ungebrochen in den neuen Staat zu überführen. Etwa die Kirchen oder die einzelstaatlichen Parlamente, Bürokratien, Parteien, Verbände, um nur einiges zu nennen. Der Föderalismus verband mithin das Neue mit der Geschichte. Die Legitimationsleistung, die darin liegt, würde der Vergleich mit Italien zeigen. Dort gab es diesen föderativen Translator nicht. Das schuf erhebliche Probleme. Der italienische National- 
staat trat als Eroberungsstaat ins Leben, der die Einzelstaaten dauerhaft ausgelöscht hat. ${ }^{35}$

- Die stärkste breitenwirksame Integrationskraft war der Erfolg. Die Dynamik des Erfolgs überwand die vielen Vorbehalte derer, die den Nationalstaat in dieser Gestalt nicht gewollt hatten. Mit Erfolg ist nicht nur dessen enorme wirtschaftliche Prosperität gemeint. Zur Integration durch Erfolg gehörten ebenso die neuen Formen sozialer Sicherung, die Verbesserung des Bildungswesens, der Ausbau des Rechtsstaates, die Machtsteigerung in der internationalen Politik ${ }^{36}$ und nicht zuletzt der Bedeutungszuwachs des Bundesparlaments und der Auf- und Ausbau von Bundesbehörden. Die Entwicklungsdynamik ging mehr und mehr von der Bundesebene aus. Auch auf Seiten der Parlamente. Hier allerdings zeigte sich die föderative Struktur nicht nur integrationsförderlich. Die Staatenkammer wirkte als Parlamentarisierungsblockade. Sie musste durchbrochen werden, um eine volle parlamentarische Regierungsform zu ermöglichen. Das war erneut eine Kriegsfolge. $\mathrm{Ob}$ es auch ohne den Ersten Weltkrieg möglich gewesen wäre, zumindest so schnell, wissen wir nicht.

Damit sei die Analogiekonstruktionen zwischen nationaler Geschichte und EUGegenwart abgebrochen - es bleibt festzuhalten: Man sollte das europäische Projekt nicht in ein Demokratie-Dilemma hineinreden, nur weil es bislang vorrangig ein Elitenprojekt ist. Historisch ist das die Normalität. Gesellschaftliche Legitimation folgt der Staatsbildung. Allerdings nicht jeder. Sie muss die Lebenswelt der Menschen verbessern, und sie muss ihnen in der Entwicklungsperspektive Partizipationsmöglichkeiten bieten, die den Erwartungen der Zeit genügen. Föderalismus in jederlei Gestalt - und es gab und gibt viele Gestalten ist dabei hilfreich, da er die Transformationen erleichtert, indem er Vertrautes in das Neue überführt. Etwa wenn Schweizer heute ihr souveränitätsbegrenzendes Sonderverhältnis zur EU mit einem „Zugewandten Ort“ der alten Eidgenossenschaft vergleichen. ${ }^{37}$

35 Scharf herausgerarbeitet (mit der Charakterisierung als Eroberungsstaat: unification by conquest) bei Ziblatt, D.: Structuring the State. The formation of Italy and Germany and the puzzle of federalism. Princeton, 2006; zur Transformationsleistung des deutschen Föderalismus über die Brüche in den Staatsordnungen hinweg s. (mit der Fachliteratur) Weichlein, S.: Föderalismus und Bundesstaat zwischen dem Alten Reich und der Bundesrepublik Deutschland, in: Handbuch Föderalismus, I, a.a.O., 101-127; Langewiesche, D.: Historische Reflexionen zum Föderalismus in Deutschland. Wandel und Kontinuität seit dem 19. Jahrhundert, ebd., 129-143.

36 Diesen Aspekt, der in den Begründungen für die Notwendigkeit eines geeinten Europa ein erhebliches Gewicht einnimmt (sei es als Wirtschaftsmacht oder als Akteur in der internationalen Politik), verfolgt im historischen Längsschnitt bis in die Gegenwart Simms, B.: Towards a mighty union: how to create a democratic European superpower, in: International Affairs 88/1 (2012), 49-62.

37 Maissen, Th.: Geschichte der Schweiz, a.a.O., 2011, 323. 
Damit zu schließen, klänge aber allzu harmonisch. Deshalb sei am Ende nochmals die Transformationskraft Krieg angesprochen. Historisch gilt: keine Staatsbildung ohne Krieg, ohne Krieg kein Übergang vom Staatenbund zum Bundesstaat. Der Krieg als Pfadbrecher und Pfadbildner. Die EU scheint dabei zu sein, um Andreas Wirschings Argumentation aufzunehmen, den Krieg als Pfadbildner und auch als vergesellschaftende Integrationskraft durch die Krise zu ersetzen. Das wäre eine weltgeschichtliche Innovation. Deshalb ist eine Rückversicherung an der Geschichte ratsam. Zu ihren Orientierungsangeboten gehören der Föderalismus und die Tradition des zusammengesetzten Staates. Aber beides war und ist in den EU-Staaten höchst unterschiedlich gewichtet. Es ist zu vermuten, dass die Tradition des zusammengesetzten Staates diesen unterschiedlichen europäischen Geschichtserfahrungen eher entspricht als der Begriff Föderalismus. Denn er privilegiert innerhalb der EU die deutsche Geschichtserfahrung und die deutsche Institutionenordnung. Das wird man ein Problem für Europa nennen dürfen. 